\title{
Analyzing the Application of Myocardial Blood Flow Index in the Diagnosis of In-Stent Stenosis
}

\author{
Qing-Feng Xiong ${ }^{1}$, Xiao-Rong $\mathrm{Fu}^{2}$, Juan $\mathrm{Xu}^{3}$ and Guang-Yao $\mathrm{Wu}^{1,4, *}$ \\ ${ }^{1}$ Department of Radiology, Zhongnan Hospital of Wuhan University, Wuhan, People's Republic of China \\ ${ }^{2}$ Department of Pediatrics, Wuchang Hospital of University of Science and Technology, Wuhan, People's Republic of China \\ ${ }^{3}$ Department of Radiology, Wuhan Asia Heart Hospital of University of Science and Technology, Wuhan, People's Republic of China \\ ${ }^{4}$ Department of Radiology, Shenzhen University General Hospital, Shenzhen University Clinical Medical Academy, Shenzhen University International Cancer Center, \\ Shenzhen, People's Republic of China \\ "Corresponding author: Department of Radiology, Zhongnan Hospital of Wuhan University, Wuhan, People’s Republic of China. Email: 2534689677@qq.com
}

Received 2020 July 11; Revised 2021 January 22; Accepted 2021 January 25.

\begin{abstract}
Background: In-stent restenosis (ISR) usually develops from stent neointimal hyperplasia (SNH), which will seriously weaken the effect of treatment. In this study, both SNH and ISR were classified as in-stent stenosis (ISS), and a non-invasive parameter - myocardial blood flow index (MBFI) was used to analyze its value in the diagnosis of stent abnormalities.

Objectives: Analyzing the application of MBFI in the diagnosis of ISS.

Patients and Methods: Clinical follow-up data for 572 patients with drug-eluting stent (DES) was collected continuously. Ninety cases were screened with the protocol of coronary computed tomography angiography (CCTA) and invasive coronary angiography (ICA) for the unrelieved symptoms. In-stent abnormalities included SNH (ISS $<50 \%$ ) and ISR(ISS $\geq 50 \%$ ). The ROC curve was analyzed using the optimal cutoff value of MBFI to evaluate the in-stent abnormalities. $t$-test of independent samples was used for the comparison data with normal distribution, and chi-square test was used for comparison of nominal variables. $\mathrm{P}<0.05$ was considered statistically significant.

Results: Frequency of ISS was not statistically different between genders $\left(\chi^{2}=0.105\right)(\mathrm{P}=0.7463)$. The optimal cutoff value was 0.082 with the area under the curve (AUC) of $0.829(\mathrm{P}<0.001)$. The sensitivity, specificity, positive predictive value, and negative predictive value were $91.4 \%, 89.1 \%, 84.2 \%$, and $94.2 \%$, respectively, and the accuracy was $90.0 \%$. Among 39 cases with MBFI $\leq 0.082,34$ $(37.8 \%, 34 / 90)$ were with ISS, including $18(20 \%, 18 / 90)$ of SNH, and $16(17.8 \%, 16 / 90)$ of ISR. In the 39 cases with MBFI $\leq 0.082$, there were eight $(20.5 \%, 8 / 39)$ presented new lesions, and seven $(17.9 \%, 7 / 39)$ presented severe lesions.

Conclusion: MBFI could be used for evaluating ISS, and more attention should be paid to the new accompanying lesions for the high risk of severe lesions.
\end{abstract}

Keywords: Coronary CT Angiography, Coronary Artery Disease, Invasive Coronary Angiography, In-Stent Stenosis, Myocardial Blood Flow Index, Diagnosis Test

\section{Background}

Using drug eluting stent (DES) is one of the most effective methods for acute myocardial infarction (1), but the effect has been seriously impaired by stent abnormalities (2). The mechanism is mediated by a series of vascular active substances, and the process could not be prevented easily $(3,4)$. Furthermore, the definite mechanism is not completely clear (5). The research by Lee et al. (6) showed that coronary artery calcification score and cardiac enzyme (CK-MB) could predict in-stent restenosis (ISR). Some scholars also noted that the distribution width of red blood cells reflecting the physiological state of the body could also predict ISR (7). Therefore, the occurrence of ISR may manifest itself in a variety of forms, and the indicators that reflect the physiological changes of the body may also reflect ISR. In this study, myocardial remodeling was used as a pathophysiological indicator (8).

\section{Objectives}

The main objective of this study was to analyze the possibility of myocardial blood flow index (MBFI) in the diagnosis of in-stent stenosis (ISS). 


\section{Patients and Methods}

\subsection{Clinical Protocols}

Cases were continuously collected.

Inclusion criteria: Clinical follow-up data for 572 patients, who had been treated with DES for acute coronary syndrome, were being collected continuously from February 2008 to May 2015. There were 90 cases suspected of ISS. This prospective study was in conformity with the declaration of Helsinki. The approval number obtained from Medical Ethics Committee of Medical College of Wuhan University of Science and Technology was 202038. All patients gave written informed consent.

Exclusion criteria is listed in Figure 1.

\subsection{Coronary Computed Tomography Angiography (CCTA) Pro-} tocol

\subsubsection{Scanning Parameters}

CCTA was performed using 256-slice multidetector computerized tomography (MDCT) scan (Brilliance, Philips Medical System, The Netherlands). The scan parameters included slice collimation $=128 \times 0.625 \mathrm{~mm}$, rotation time $=0.27 \mathrm{~s}$, tube current-time product $=125 \mathrm{mAs}$, tube potential $=120 \mathrm{kVp}$ and resulting CTDIvol $=10.5 \mathrm{mGy}$. The prospective electrocardiogram (ECG) gating scanner was used. Scanning was carried out at the end of deep inspiration. Based on the patient's body weight, a bolus of 50 to $65 \mathrm{~mL}$ contrast media (Ultravist $370 \mathrm{mg}$ iodine/mL, Bayer) was injected into the antecubital vein at the rate of 3.8 - 4.3 $\mathrm{mL} / \mathrm{s}$, followed by a $30 \mathrm{ml}$ bolus of saline solution chaser through a dual-head injector (Irich medical, Missouri XD2001, Germany). An automated bolus tracking method was used for triggering when the attenuation reached 120 $\mathrm{HU}$ in the region of interest of the ascending aorta with a 6 seconds delay. Prospective ECG-triggered sequential acquisition mode was performed in all patients (with the acquisition window of $30 \%-85 \%$ of the R-R interval). The maximum intensity projection (MIP), multi-plane reconstruction (MPR) and curved planar reconstructions (CPR) imaging were used to analyze.

\subsubsection{CT Image Reconstruction}

We reconstructed axial source images with a section thickness of $0.67 \mathrm{~mm}$ and a section interval of $0.33 \mathrm{~mm}$ by using a standard filtered back projection (FBP) algorithm with a high-resolution cardiac kernel/filter during the middiastolic phase. The center of the imaging window was set at $75 \%$ of the R-R interval.

\subsubsection{Image Quality Assessment}

Image quality was assessed by two senior specialists with 10 years working experience in CCTA, and the disagreement was resolved by discussing. Excellent images without artifacts or pixel noises were selected (9).

\subsubsection{Data Analysis of CCTA}

The visible lumen diameter was given as an average of the measurements on three representative axial slices using the electronic caliper tool provided with the scanner's standard workstation (IntelliSpace Portal, Philips Healthcare) (10). The length and inner lumen diameter of the stents (including from Abbott Co, US, Boston Scientific Co, US, and Medtronic Inc, US) were $26 \mathrm{~mm}-30 \mathrm{~mm}$ and $2.8 \mathrm{~mm}$ - 3.5mm, respectively. The stent materials included cobalt and chromium alloy, platinum and chromium alloy, cobalt and nickel alloy, with the wall thickness less than $0.1 \mathrm{~mm}$ and the profile of $0.95 \mathrm{~mm}-1.19 \mathrm{~mm}$. The drug film was sirolimus (Cypher, Jihnson \& Johnson, USA).

Coronary artery plaque and stenosis were assessed by the fifteen-segment method (11). The new progressive plaque was judged by comparing with the result of previous invasive coronary angiography (ICA) before. When the stenosis was more obvious than before, it was defined as a new lesion.

\subsection{Invasive Coronary Angiography Protocol}

Invasive coronary angiography (ICA) was performed within 1 week after admission. The angiograms were reported by experienced invasive cardiologists blinded to the CCTA results. The ICA results were classified as follows: no stenosis; stenosis < 50\%; 50\% $\leq$ stenosis $<70 \%$; 70\% $\leq$ stenosis $<99 \%$; and occlusion. Stenosis of the stent was classified as ISR with the lumen reduction $\geq 50 \%$, or as stent neo-intimal hyperplasia $(\mathrm{SNH})$ with the lumen reduction $<50 \%$. The morphology of stent was evaluated by the literature (12), including focal stenosis $<10 \mathrm{~mm}$ in the stent with a low density filling defect; diffuse stenosis $\geq 10 \mathrm{~mm}$ in the stent with a low density filling defect; proliferative stenosis $>10 \mathrm{~mm}$ and extending outside the either end of the stent with a low density filling defect; occlusion.

\subsection{Myocardial Blood Flow Index (MBFI)}

\subsubsection{Myocardial Blood Flow Model}

According to the lumped parameter network (LPN) (13), myocardial perfusion was assumed to be a parallel impedance model (14), and myocardial blood flow was mainly determined by the ratio of myocardial blood perfusion pressure to myocardial microcirculation resistance 


\section{There were 572 sequential acute coronary syndrome patients who underwent revascularization therapy}

The patients were excluded before the protocol of coronary CT angiography (CCTA) was performed, including 101 with acute myocardial infarction with ST-segment elevation, 2 with allergy to iodine, 3 with breathlessness, 1 with obvious arrhythmia, 47 with heart rate less than 60 beats/min or more than 85 beats/min, 28 with severe impairment of renal function (glomerular filtration rate $<30 \mathrm{~mL} / \mathrm{min} / 1.73 \mathrm{~m} 2,3$ with spastic bronchial asthma, 94 with old myocardial infarction and a ventricular aneurysm, 18 with coronary artery bypass graft, 13 with II-III degree atrioventricular block, and 11 lost to follow-up.

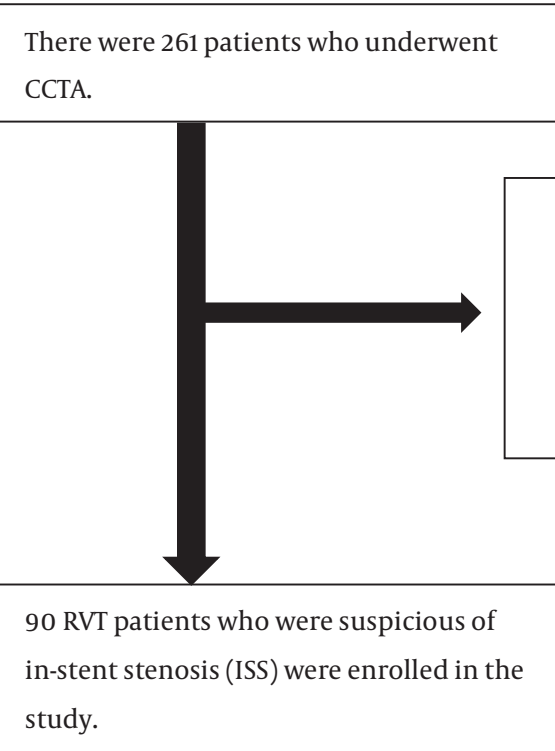

Figure 1. Patient enrollment flow chart

The patients were excluded after the protocol of CCTA was performed, including 28 younger than 30 years old or older than 75 years old, 5 with valvular disease, 6 with myocardial hypertrophy in hypertensive patients, 1 with constrictive pericarditis, 3 with hypothyroidism, 118 with imperfect image quality,13 with II-III degree artrioventricular block

(myocardial mass) (15). Since the cardiac blood circulation is pulsed from the artery end to the vein end in about five cardiac cycles (16), that is, after five cardiac cycles, a certain quantity of blood is distributed to the whole cardiac microcirculation. Therefore, the ratio of myocardial blood flow quantity to the mass is the myocardial blood flow (Figure 2). Because body mass index (BMI) (17), gender (18), and age (19) all correlate with coronary artery disease, so MBFI should be corrected.

\subsubsection{Calculation of $M B F I$}

Using Omron upper-arm blood pressure monitors (Omron U10K, Dalian, China), the patients' diastolic blood pressures (DP) were measured in a quiet condition, and the heart rates (HR) were recorded three times after a 10- 


\section{Myocardial Blood Flow Model}

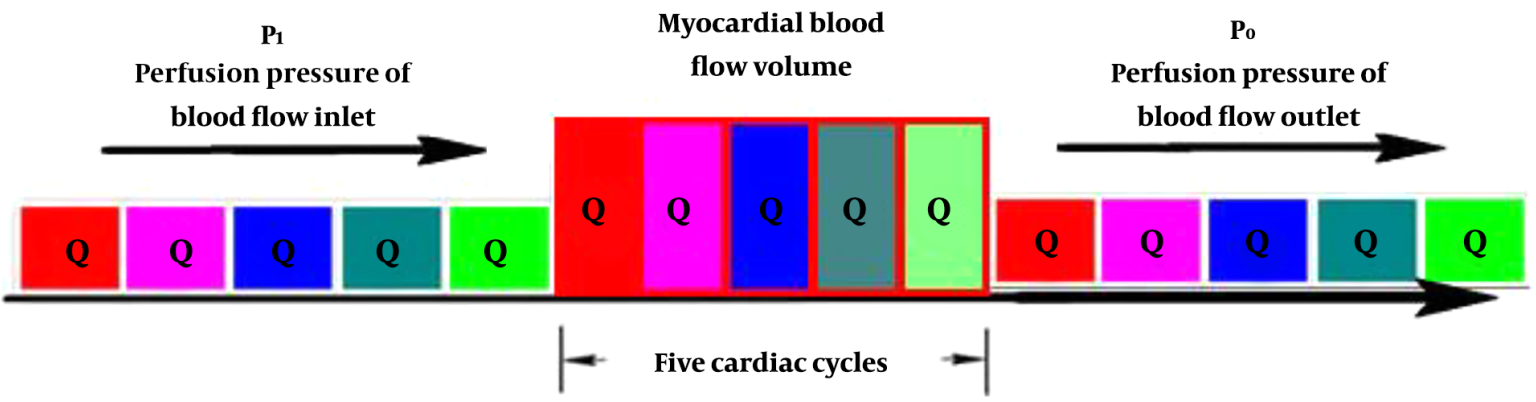

Figure 2. Myocardial blood flow model

minute interval in the sitting position. Then, using the post-processing software, the myocardial mass (M) was calculated. The cardiac cycle (time, T) was calculated as 60 seconds divided by HR within one minute. The myocardial perfusion time (PT) was equal to 5 multiplied by T. As a result, MBFI = PT multiplied by DP/ (M multiplied by BMI) (14, $16,20)$.

\subsection{Diagnostic Analysis}

ICA was used to define the degree of ISS: SNH with ISS < $50 \%$, and ISR with ISS $\geq 50 \%$. The receiver operating characteristic (ROC) curve was analyzed by 2 times the standard deviation of the mean, and the optimal cutoff value of MBFI was determined to diagnose the stent abnormality. The diagnosis accuracy was analyzed, including the sensitivity, specificity, positive predictive value, and negative predictive value.

\subsection{Statistical Analysis}

Statistical analyses were performed using commercially available software (MedCalc Software 18.2.1, Ostend, Belgium). Quantitative variables were expressed as mean \pm standard deviation(SD), and categorical data were given in proportions and percentages. Continuous variables were compared using $t$ test, whereas count data were compared using chi-square test. $\mathrm{P}<0.05$ was considered statistically significant.

Multivariate regression analysis was performed to obtain the weight coefficient of the old age. MBFI and old age were assumed to be high risk factors for ISS. Old age was considered as higher or equal to 60 years for male or higher or equal to 55 years for female. The age intervals were set according to an interval of 5 years. The contribution of old age in the model was multiplying the intervals by the weight coefficient of age.

\section{Results}

Among the 90 patients (mean age, $62.6 \pm 10.02$ years), the time of stent implantation was $7.1 \pm 4.51$ years. Baseline characteristics are shown in Table1. MBFI was the main risk factors of ISS $(\mathrm{P}<0.0001)$. The weight coefficient of the old age was 0.01. Corrected MBFI = MBFI -k multiplied by $\mathrm{n}(\mathrm{k}=0.01$ (the weight coefficient)), $\mathrm{n}=$ age intervals. Two times the standard deviation of the mean value was 0.100 , and the optimal cut-off value was 0.082 (area under the curve $[\mathrm{AUC}]=0.829,95 \% \mathrm{CI}: 0.736-0.900$ ) (Figure 3 ). There were 39 cases with MBFI $\leq 0.082$, of which $34(37.8 \%$, $34 / 90)$ showed ISS, including 18 (20\%, 18/90) with SNH, 16 $(17.8 \%, 16 / 90)$ with ISR (Figure 4$)$. In the 39 cases with MBFI $\leq 0.082,8$ (20.5\%, 8/39) presented new lesions, and 7 (17.9\%, 7/39) presented severe lesions. There were 51 cases with MBFI > 0.082, and only one case with SNH (Figure 5). The sensitivity, specificity, positive predictive value, and negative predictive value were $91.4 \%, 89.1 \%, 84.2 \%$, and $94.2 \%$, respectively, and the accuracy was $90.0 \%$.

\section{Discussion}

Cardiac remodeling, as an important aspect of pathological changes in coronary artery disease (CAD) (21), would be an important breakthrough point for the study of ISR. In this study, with the ICA as the gold standard, among all 39 cases with $\mathrm{MBFI} \leq 0.082$, there were 34 cases 


\begin{tabular}{|c|c|c|c|c|}
\hline Characteristics & Male $(n=59)$ & Female $(n=31)$ & tor $\chi^{2}$ value & $\mathbf{P}$ \\
\hline Age, $y$ & $64.8 \pm 9.62$ & $63.6 \pm 8.32$ & -0.588 & 0.5579 \\
\hline BMI, $\mathrm{kg} / \mathrm{m}^{2}$ & $24.2 \pm 4.12$ & $25.2 \pm 2.69$ & 1.220 & 0.2257 \\
\hline Heart rate, times/min & $71.7 \pm 10.10$ & $70.5 \pm 10.02$ & -0.537 & 0.5926 \\
\hline Diastolic pressure, $\mathbf{m m H g}$ & $77.8 \pm 11.12$ & $75.3 \pm 13.15$ & -0.951 & 0.3442 \\
\hline Perfusion time, $s$ & $4.2 \pm 0.79$ & $4.3 \pm 0.55$ & 0.629 & 0.5313 \\
\hline Time of stent implantation, $y$ & $7.5 \pm 4.23$ & $7.1 \pm 4.55$ & -0.415 & 0.6789 \\
\hline In-stent stenosis, $\mathbf{n}$ & 23 & 11 & 0.105 & 0.7463 \\
\hline
\end{tabular}

Abbreviation: BMI, body mass index.

${ }^{\mathrm{a}}$ Values are expressed as mean $\pm \mathrm{SD}$.

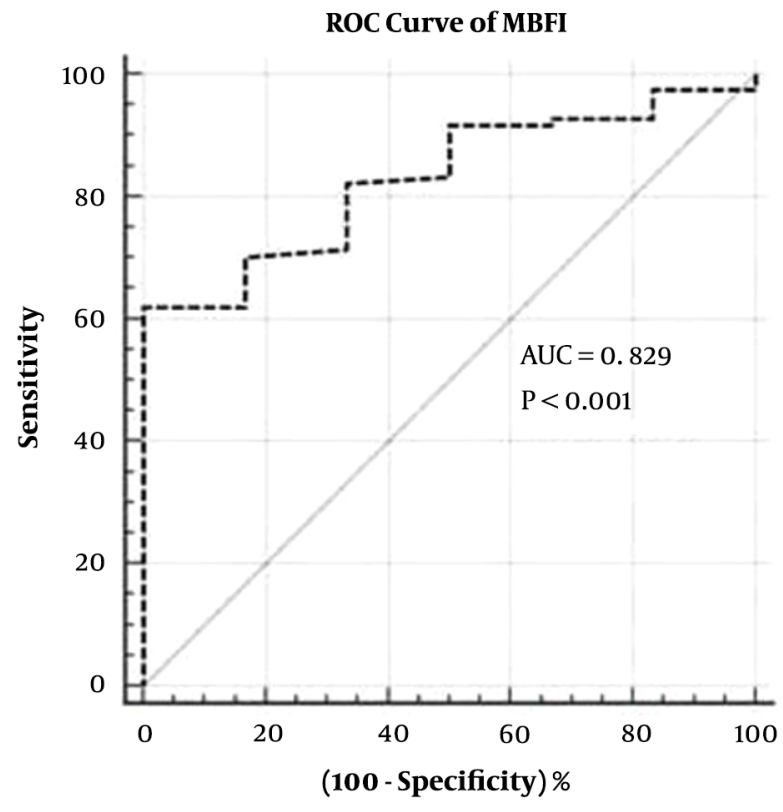

Figure 3. ROC curve analysis of MBFI

with ISS (37.8\%, 34/90), including 18 cases $(20.0 \%, 18 / 90)$ of SNH, 16 cases $(17.8 \%, 16 / 90)$ of ISR, which was higher than the literature $(<10 \%)(22)$. There were a few reasons for the higher incidence rate of ISR including enrolled cases with the vast majority of older patients $(75.6 \%, 68 / 90)$, the relative long time after stent implantation (up to 13 years), and the different type of DES with the inconsistent efficacy to prevent ISS (23). It can be seen that stent abnormalities include SNH and ISR (24), which can be analyzed by MBFI. To know whether MBFI can further differentiate SNH from ISR directly, further studies are necessary.

For CCTA, evaluating ISS is susceptible to some factors, such as metal artifacts, respiratory movement artifacts, arrhythmia, and the limited spatial resolution. It could be difficult for CCTA to evaluate ISS accurately. If a special tool is used in the diagnosis of ISS, the accuracy would improve obviously. This study used MBFI to evaluate ISS without thinking about the weakness of CCTA, and found out that the accuracy was satisfied with MBFI, and the sensitivity, specificity, positive predictive value, negative predictive value were $91.4 \%, 89.1 \%, 84.2 \%$, 94.2\% respectively, and the accuracy was $90 \%$. The introduction of MBFI to analyze stent abnormalities in the pathophysiological perspective of remodeling could avoid the method of conventional deficiency based on the morphology of the stent, and provide an objective basis to prevent ISR in a high-risk population with an ISS lower than $50 \%$.

After stent implantation, patients were treated with dual-antiplatelet therapy (aspirin combined with clopidogrel) and secondary prevention of CAD in accordance with relevant guidelines (25), and the plaque of the coronary artery should be stable or improved. However, in this study, in the 39 cases with MBFI $\leq 0.082,20.5 \%(8 / 39)$ were found with the new-onset plaque and $17.9 \%$ (7/39) presented with severe stenosis after stent implantation. The reasons could be remodeling factors including cytokines inducing smooth muscle cell differentiation, accelerating cell synthesis, and promoting proliferation and migration, which could induce the occurrence of the unstable atherosclerotic plaque. They could be the potential risk factors leading to acute coronary syndrome and great attention should be paid to them in clinical practice.

This study has limitations. First, this study chose a resting state in a relatively short period of one day, so further research is needed to determine which time is more representative. Second, similar to the risk factors of the elderly, multi-stent implantation, diabetes and multi-vessel 

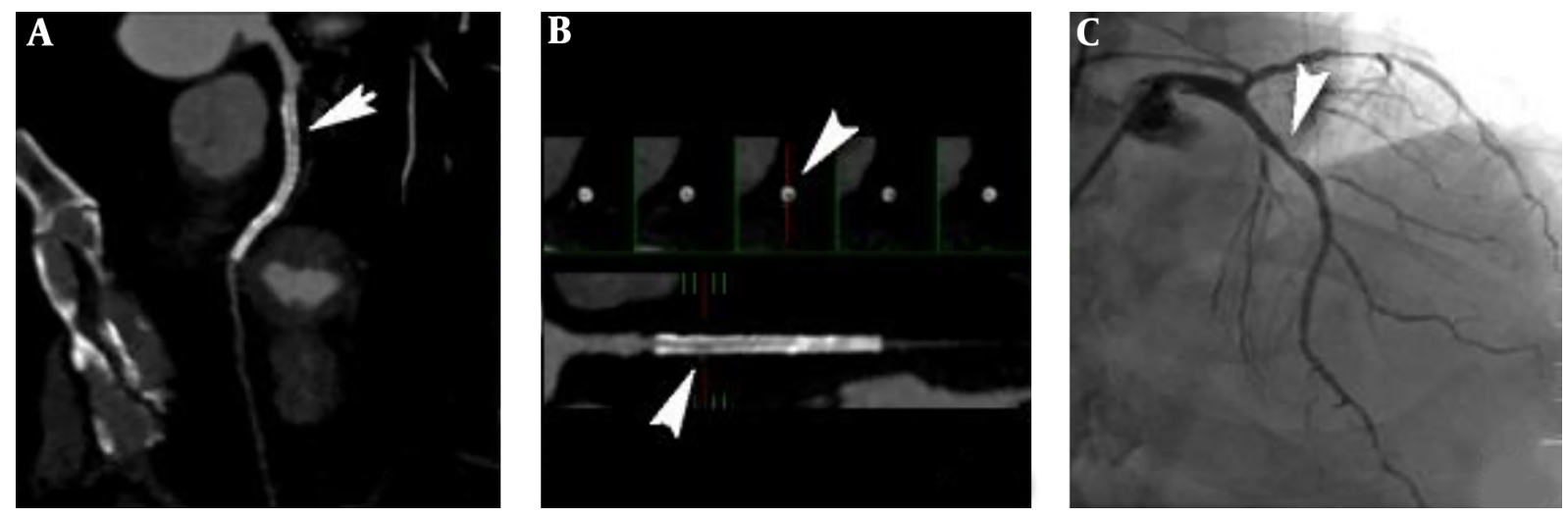

Figure 4. A 70-year-old female patient with unstable angina, left anterior descending (LAD) artery, and two drug eluding stents (DES) for the diffuse severe lesions. A and B, Computed coronary tomography angiography (CCTA) shows an in-stent restenosis (ISR) (arrows, window level 486, window width 843) in the LAD artery with the myocardial blood flow index (MBFI) of 0.055 ; C, Invasive coronary angiography (ICA) shows a stenosis degree of $70 \%$ in the stent (arrow).
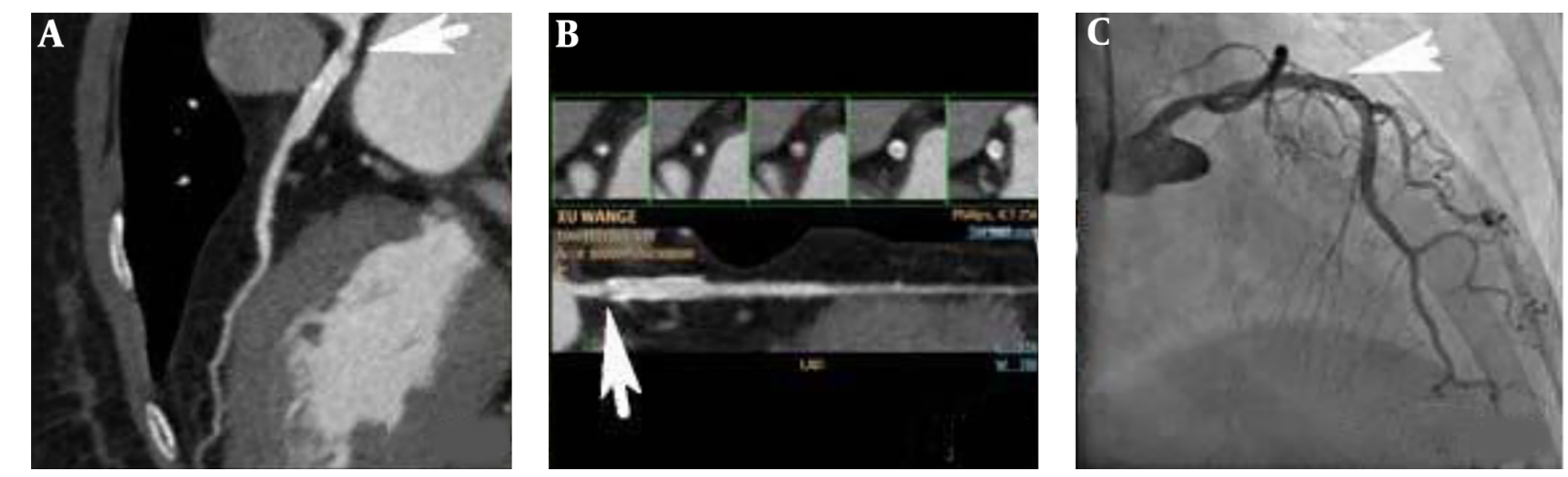

Figure 5. A 78-year-old male patient with unstable angina, left anterior descending (LAD) artery, and a drug eluding stent (DES) for the local severe lesion. A and B, Computed coronary tomography angiography (CCTA) shows a small low-density shadow (arrows, window level 453, window width 872) in the proximal segment of stent with the myocardial blood flow index (MBFI) of $0.089 ; \mathrm{C}$, Invasive coronary angiography (ICA) does not show in-stent stenosis (ISS) in the stent (arrow).

lesions are also risk factors of ISS, so it is necessary to further explore their roles in the model.

In conclusion, all patients with reduced MBFI values after stent implantation should be actively prevented from the occurrence of ISR, and ICA examination should be performed if necessary. Second, ISS could accompany new severe lesions leading to acute coronary syndrome, to which great attention should be paid in clinical practice.

\section{Footnotes}

Authors' Contributions: Study concept and design: QX, and GW. Analysis and interpretation of data: QX, and GW. Drafting of the manuscript: XF. Critical revision of the manuscript for important intellectual content: QX, JX, and GW. Statistical analysis: JX.
Clinical Trial Registration Code: The study has been registered in China clinical trial registry (no.: chictr180018376).

Conflict of Interests: The authors declare that there is no conflict of interests regarding the publication of this paper.

Ethical Approval: No.: 202038 from the institutional review board of Medical Ethics Committee of Medical College of Wuhan University of Science and Technology, Wuhan, China.

Funding/Support: This study was supported in part by grant WX18C01 from the Medical research project of Wuhan Health Committee.

Informed Consent: All patients gave written informed consent. 


\section{References}

1. Eisenstein EL, Anstrom KJ, Kong DF, Shaw LK, Tuttle RH, Mark DB, et al. Clopidogrel use and long-term clinical outcomes after drug-eluting stent implantation. JAMA. 2007;297(2):159-68. doi: 10.1001/jama.297.2.joc60179. [PubMed: 17148711].

2. Tocci G, Barbato E, Coluccia R, Modestino A, Pagliaro B, Mastromarino $\mathrm{V}$, et al. Blood Pressure Levels at the Time of Percutaneous Coronary Revascularization and Risk of Coronary In-Stent Restenosis. Am J Hypertens. 2016;29(4):509-18. doi: 10.1093/ajh/hpv131. [PubMed: 26271109]. [PubMed Central: PMC4886488].

3. Akboga MK, Yalcin R, Sahinarslan A, Yilmaz Demirtas C, Abaci A. Effect of serum YKL-40 on coronary collateral development and SYNTAX score in stable coronary artery disease. Int J Cardiol. 2016;224:323-7. doi: 10.1016/j.ijcard.2016.09.042. [PubMed: 27668705].

4. Yilmaz S, Akboga MK, Aras D, Topaloglu S. Evaluation of the Predictive Value of CHA2DS2-VASc Score for In-Stent Restenosis. Angiology. 2018;69(1):38-42. doi: 10.1177/0003319717700746. [PubMed: 28345395].

5. Akboga MK, Yilmaz S. Predictors of In-Stent Restenosis. Angiology. 2019;70(3):279. doi: 10.1177/0003319718776796. [PubMed: 29783847].

6. Lee JB, Choi YS, Chung WB, Kwon A, Park CS, Lee MY. High coronary calcium score and post-procedural CK-MB are noninvasive predictors of coronary stent restenosis. Clin Interv Aging. 2017;12:399404. doi: 10.2147/CIA.S125592. [PubMed: 28255235]. [PubMed Central: PMC5322937].

7. Qian H, Luo Z, Xiao C, Chen J, Li D, Xu H, et al. Red cell distribution width in coronary heart disease: prediction of restenosis and its relationship with inflammatory markers and lipids. Postgrad Med J. 2018;94(1115):489-94. doi: 10.1136/postgradmedj-2018-135806. [PubMed: 30301834].

8. Xiong QF, Ma XJ, Chen Y, et al. Clinical significance of myocardial blood flow index derived from CCTA in the diagnosis of calcified plaque-related coronary artery disease.J Radiol Pract. 2019;(18):1327-31.

9. Liu Y, Li J, Zhao H, Jia Y, Ren J, Xu J, et al. Image quality and radiation dose of dual-source CT cardiac angiography using prospective ECG-triggering technique in pediatric patients with congenital heart disease. J Cardiothorac Surg. 2016;11:47. doi: 10.1186/s13019-016-0460-9. [PubMed: 27059600]. [PubMed Central: PMC4826524].

10. Hickethier T, Wenning J, Doerner J, Maintz D, Michels G, Bunck AC. Fourth update on CT angiography of coronary stents: in vitro evaluation of 24 novel stent types. Acta Radiol. 2018;59(9):1060-5. doi: 10.1177/0284185117744227. [PubMed: 29181989].

11. Austen WG, Edwards JE, Frye RL, Gensini GG, Gott VL, Griffith LS, et al. A reporting system on patients evaluated for coronary artery disease. Report of the Ad Hoc Committee for Grading of Coronary Artery Disease, Council on Cardiovascular Surgery, American Heart Association. Circulation. 1975;51(4 Suppl):5-40. doi: 10.1161/01.cir.51.4.5. [PubMed: 1116248].

12. Slavin L, Chhabra A, Tobis JM. Drug-eluting stents: preventing restenosis. Cardiol Rev. 2007;15(1):1-12. doi: 10.1097/01.crd.0000200844.16899.fc. [PubMed: 17172878].

13. Sinclair MD, Lee J, Cookson AN, Rivolo S, Hyde ER, Smith NP. Measurement and modeling of coronary blood flow. Wiley Interdiscip Rev Syst Biol Med. 2015;7(6):335-56. doi: 10.1002/wsbm.1309. [PubMed: 26123867].
14. Westerhof N, Bosman F, De Vries CJ, Noordergraaf A. Analog studies of the human systemic arterial tree. J Biomech. 1969;2(2):121-43. doi: 10.1016/0021-9290(69)90024-4. [PubMed: 16335097].

15. Hamada M, Kuwahara T, Shigematsu Y, Kodama K, Hara Y, Hashida $\mathrm{H}$, et al. Relation between coronary blood flow and left ventricular mass in hypertension: noninvasive quantification of coronary blood flow by thallium-201 myocardial scintigraphy. Hypertens Res. 1998;21(4):227-34. doi: 10.1291/hypres.21.227. [PubMed: 9877515].

16. Lindner JR, Villanueva FS, Dent JM, Wei K, Sklenar J, Kaul S. Assessment of resting perfusion with myocardial contrast echocardiography: theoretical and practical considerations. Am Heart J. 2000;139(2 Pt 1):231-40. [PubMed:10650295].

17. Dores H, de Araujo Goncalves P, Carvalho MS, Sousa PJ, Ferreira A, Cardim N, et al. Body mass index as a predictor of the presence but not the severity of coronary artery disease evaluated by cardiac computed tomography. EurJ Prev Cardiol. 2014;21(11):1387-93. doi: 10.1177/2047487313494291. [PubMed: 23774273].

18. Yahagi K, Davis HR, Arbustini E, Virmani R. Sex differences in coronary artery disease: pathological observations. Atherosclerosis. 2015;239(1):260-7. doi: 10.1016/j.atherosclerosis.2015.01.017. [PubMed: 25634157].

19. Gurel NZ, Carek AM, Inan OT, Levantsevych O, Abdelhadi N, Hammadah $\mathrm{M}$, et al. Comparison of autonomic stress reactivity in young healthy versus aging subjects with heart disease. PLoS One. 2019;14(5). e0216278. doi: 10.1371/journal.pone.0216278. [PubMed: 31067240]. [PubMed Central: PMC6505888].

20. Artru AA, Katz RA, Colley PS. Autoregulation of cerebral blood flow during normocapnia and hypocapnia in dogs. Anesthesiology. 1989;70(2):288-92. doi: 10.1097/00000542-198902000-00018. [PubMed: 2492410].

21. Xue $X$, Luo L. LncRNA HIF1A-AS1 contributes to ventricular remodeling after myocardial ischemia/reperfusion injury by adsorption of microRNA-204 to regulating SOCS2 expression. Cell Cycle. 2019;18(19):2465-80. doi: 10.1080/15384101.2019.1648960. [PubMed: 31354024]. [PubMed Central: PMC6739046].

22. Colombo A, Drzewiecki J, Banning A, Grube E, Hauptmann K, Silber S, et al. Randomized study to assess the effectiveness of slow- and moderate-release polymer-based paclitaxel-eluting stents for coronary artery lesions. Circulation. 2003;108(7):788-94. doi: 10.1161/01.CIR.0000086926.62288.A6. [PubMed: 12900339].

23. Sabate M, Jimenez-Quevedo P, Angiolillo DJ, Gomez-Hospital JA, Alfonso F, Hernandez-Antolin R, et al. Randomized comparison of sirolimus-eluting stent versus standard stent for percutaneous coronary revascularization in diabetic patients: the diabetes and sirolimus-eluting stent (DIABETES) trial. Circulation. 2005;112(14):2175-83. doi: 10.1161/CIRCULATIONAHA.105.562421. [PubMed: 16203930].

24. Jensen LO, Vikman S, Antonsen L, Kosonen P, Niemela M, Christiansen $\mathrm{EH}$, et al. Intravascular ultrasound assessment of minimum lumen area and intimal hyperplasia in in-stent restenosis after drug-eluting or bare-metal stent implantation. The Nordic Intravascular Ultrasound Study (NIVUS). Cardiovasc Revasc Med. 2017;18(8):577-82. doi: 10.1016/j.carrev.2017.05.010. [PubMed: 29066343].

25. Neumann F, Sousa-Uva M, Ahlsson A, Alfonso F, Banning AP, Benedetto U, et al. 2018 ESC/EACTS Guidelines on myocardial revascularization. Eur Heart J. 2019;40(2):87-165. doi: 10.1093/eurheartj/ehy394. 